ACCEPTED MANUSCRIPT

\title{
Evidence of precursor orthorhombic domains well above the electronic nematic transition temperature in $\operatorname{Sr}($ Fe $<$ sub $>1-x</$ sub $>$ Co $<$ sub $>$ x $</$ sub $>$ ) $<$ sub $>2</$ sub $>$ As $<$ sub $>2</$ sub $>$
}

To cite this article before publication: Ulisses Kaneko et al 2019 J. Phys.: Condens. Matter in press https://doi.org/10.1088/1361-648X/ab2ffC

\section{Manuscript version: Accepted Manuscript}

Accepted Manuscript is "the version of the article accepted for publication including all changes made as a result of the peer review process, and which may also include the addition to the article by IOP Publishing of a header, an article ID, a cover sheet and/or an 'Accepted Manuscript' watermark, but excluding any other editing, typesetting or other changes made by IOP Publishing and/or its licensors"

This Accepted Manuscript is @ 2019 IOP Publishing Ltd.

During the embargo period (the 12 month period from the publication of the Version of Record of this article), the Accepted Manuscript is fully protected by copyright and cannot be reused or reposted elsewhere.

As the Version of Record of this article is going to be / has been published on a subscription basis, this Accepted Manuscript is available for reuse under a CC BY-NC-ND 3.0 licence after the 12 month embargo period.

After the embargo period, everyone is permitted to use copy and redistribute this article for non-commercial purposes only, provided that they adhere to all the terms of the licence https://creativecommons.org/licences/by-nc-nd/3.0

Although reasonable endeavours have been taken to obtain all necessary permissions from third parties to include their copyrighted content within this article, their full citation and copyright line may not be present in this Accepted Manuscript version. Before using any content from this article, please refer to the Version of Record on IOPscience once published for full citation and copyright details, as permissions will likely be required. All third party content is fully copyright protected, unless specifically stated otherwise in the figure caption in the Version of Record.

View the article online for updates and enhancements. 


\title{
Evidence of precursor orthorhombic domains well above the electronic nematic transition temperature in $\operatorname{Sr}\left(\mathrm{Fe}_{1-x} \mathrm{Co}_{x}\right){ }_{2} \mathrm{As}_{2}$
}

\author{
U. F. Kaneko, ${ }^{1}$ M. M. Piva,${ }^{2}$ C. B. R. Jesus ${ }^{*},{ }^{2}$ M. E. Saleta ${ }^{\dagger},{ }^{2}$ R. R. Urbano, ${ }^{2}$ P. G. Pagliuso, ${ }^{2}$ and E. Granado ${ }^{2}$ \\ ${ }^{1}$ Brazilian Synchrotron Light Laboratory (LNLS), \\ Brazilian Center for Research in Energy and Materials (CNPEM), Campinas, São Paulo 13083-970, B̂razil \\ 2 "Gleb Wataghin" Institute of Physics, University of Campinas - UNICAMP, Campinas, São Paulo 13083-859, Brazil
}

\begin{abstract}
Raman scattering, synchrotron $\mathrm{x}$-ray diffraction, specific heat, resistivity and magnetic susceptibility measurements were performed in $\operatorname{Sr}\left(\mathrm{Fe}_{1-x} \mathrm{Co}_{x}\right)_{2} \mathrm{As}_{2}[x=0.24(3)]$ single crystals with superconducting critical temperature $T_{c}=22 \mathrm{~K}$ and two additional transitions at 132 and $152 \mathrm{~K}$ observed in both specific heat and resistivity data. A quasielastic Raman signal with $B_{2 g}$ symmetry (tetragonal cell) associated with electronic nematic fluctuations is observed. Crucially, this signal shows maximum intensity at $T_{n e m} \sim 132 \mathrm{~K}$, marking the nematic transition temperature. X-ray diffraction shows evidence of coexisting orthorhombic and tetragonal domains between $T_{n e m}$ and $T_{o} \sim 152 \mathrm{~K}$, implying that precursor orthorhombic domains emerge over an extended temperature range above $T_{n e m}$. While the height of the quasielastic Raman peak is insensitive to $T_{o}$, the temperature-dependence of the average nematic fluctuation rate indicates a slowing down of the nematic fluctuations inside the precursor orthorhombic domains. These results are analogous to those previously reported for the LaFeAsO parent oxypnictide [U.F. Kaneko et al., Phys. Rev. B 96, $014506(2017)]$. We propose a scenario where the precursor orthorhombic phase may be generated within the electronically disordered regime $\left(T>T_{n e m}\right)$ as long as the nematic fluctuation rate is sufficiently small in comparison to the optical phonon frequency range. In this regime, the local atomic structure responds adiabatically to the electronic nematic fluctuations, creating a net of orthorhombic clusters that, albeit dynamical for $T>T_{n e m}$, may be sufficiently dense to sustain long-range phase coherence in a diffraction process up to $T_{0}$.
\end{abstract}

\section{INTRODUCTION}

Iron based superconductors (FeSCs) have been largely investigated since superconductivity was found in doped LaFeAsO oxypnictides [1]. The superconductor state was also achieved in other FeSCs as in the 122 system $\mathrm{AFe}_{2} \mathrm{As}_{2}(\mathrm{~A}=\mathrm{Ca}, \mathrm{Sr}, \mathrm{Ba})$ [2-6] under chemical substitution or external applied pressure, whereas $\alpha$-FeSe [7] and $\mathrm{LiFeAs}$ have superconducting critical temperatures at $T_{c} \sim 8.5 \mathrm{~K}$ and $18 \mathrm{~K}$, respectively, without long range magnetic order. Most oxypnictides and 122 systems exhibit a tetragonal-orthorhombic transition at $T_{o}$ and an stripe spin density wave antiferromagnetic ( $\mathrm{AF}$ ) ordering at $T_{A F} \leq T_{o}$. High electronic anisotropies in the $a b$-plane also point to an electronic nematic charge stripe phase associated with Fe $t_{2 g}$ orbital differentiation [8-13], with a critical temperature $T_{n e m}$ that is normally considered to be $T_{n e m}=T_{o}$. Electronic nematic fluctuations may play an important role in the mechanism of superconductivity $[8,14]$.

Undoped 122-compounds show nearly coincident $T_{A F}$ and $T_{o}[5,15]$, whereas well separated transition temperatures are observed in other series, such as in $\mathrm{Ba}\left(\mathrm{Fe}_{1-x} \mathrm{Co}_{x}\right)_{2} \mathrm{As}_{2}$ [16-18], $\mathrm{Sr}\left(\mathrm{Fe}_{1-x} \mathrm{Co}_{x}\right)_{2} \mathrm{As}_{2}$ [42-44, $50], \mathrm{NaFe}_{1-x} \mathrm{~T}_{x} \mathrm{As}(\mathrm{T}=\mathrm{Co}, \mathrm{Rh})[19,20]$ and even in par-

* Present address: Programa de Pós-Graduação em Física, Campus Prof. José Aluísio de Campos, UFS, São Cristóvão, Sergipe 49100000, Brazil

†Present address: CONICET - Centro Atómico Bariloche, CNEA, (8400) San Carlos de Bariloche, Rio Negro, Argentina. ent oxypnictides such as $\mathrm{LnFeAsO}(\mathrm{Ln}=\mathrm{La}, \mathrm{Ce}, \mathrm{Pr}, \mathrm{Nd})$ [21-26]. So far, the origin of this large separation in some cases and its possible relation with lattice disorder has not been fully understood.

Raman spectroscopy measurements reveal a quasielastic peak $(\mathrm{QEP})$ in $\mathrm{B}_{2 g}$ symmetry $(2 \mathrm{Fe}$ cell) in several compounds as $A\left(\mathrm{Fe}_{1-x} \mathrm{Co}_{x}\right)_{2} \mathrm{As}_{2}(A=\mathrm{Ca}, \mathrm{Sr}, \mathrm{Ba}$, and Eu) [27-32], $\mathrm{Ba}_{1-p} \mathrm{~K}_{p} \mathrm{Fe}_{2} \mathrm{As}_{2}$ [33, 34], LaFeAsO [35], FeSe $[36,37]$ and $\mathrm{NaFe}_{1-x} \mathrm{Co}_{x} \mathrm{As}[19]$. The detailed origin of such signal has been interpreted either as charge/orbital nematic fluctuations in Refs. 29, 30,32, 36, 38 or spin nematic fluctuations in Refs. 31, 33, 39-41, whereas both scenarios were considered in Ref. 35. In any case, it is well established that the QEP signal is generated by electronic nematic fluctuations, being thus expected to show maximum intensity at the nematic transition temperature $T_{n e m}$. Indeed, it has been observed that the QEP shows maximum intensity at $T_{n e m} \sim T_{o}$ for compounds with nearly identical or slightly separated magnetic and structural transition temperatures [29, 31], consistent with an association of $T_{o}$ with the onset of the nematic phase. On the other hand, for $\mathrm{LaFeAsO}$ with larger splitting between $T_{A F}$ and $T_{o}$, the maximum QEP intensity was found near $T_{A F}$ [35]. This study also revealed slower nematic fluctuations in $\mathrm{LaFeAsO}$ with respect to 122 parent compounds at comparable temperatures, which has been identified as a possible cause for the larger $T_{A F} / T_{o}$ separation of the former compound [35]. Motivated by these intriguing results in $\mathrm{LaFeAsO}$, we investigate here the temperature dependence of the Raman QEP in $\operatorname{Sr}\left(\mathrm{Fe}_{1-x} \mathrm{Co}_{x}\right)_{2} \mathrm{As}_{2}$ (SFCA) belonging to the 122 system. We show that the main features previously found 
in LaFeAsO are also observed in SFCA, namely a Raman QEP with maximum intensity near $T_{n e m}<<T_{o}$ and a reduction of the nematic relaxation rate below $T_{o}$. These similar results for two materials belonging to different structural systems (122 and 1111) provide robust evidence that the onset of the orthorhombic phase in these compounds (both with $T_{o}>>T_{n e m}$ ) is not an electronic nematic transition, rather signaling the presence of a precursor electronically disordered orthorhombic phase with slower nematic fluctuations with respect to the tetragonal state. The possible factors favoring the appearance of this precursor phase in specific pnictide materials are discussed.

\section{EXPERIMENTAL DETAILS}

Single crystals of $\mathrm{Sr}\left(\mathrm{Fe}_{1-x} \mathrm{Co}_{x}\right)_{2} \mathrm{As}_{2}$ (SFCA) were synthesized through In flux method as described elsewhere [5]. The samples are in the form of thin plates with dimensions of $\sim 1 \mathrm{~mm}$ in the $a b$ plane. The Co-content of the SFCA sample is $x=0.24(3)$, determined using an energy dispersive spectroscopy (EDS) microprobe. The reported Co content is an average of the values obtained on one side of the sample at several spots of the crystal surface as suggested by the error in $x$. Such relatively large value of $x$ might seem at first sight inconsistent with the presence of magnetic ordering and structural phase transitions (see below), considering previous studies in this family [42-44]. However, it is well known that the detailed phase diagrams of Fe pnictides may depend sensibly on the detailed growth procedure [5]. Specific heat measurements were performed in a small mass calorimeter using the thermal relaxation technique. The in-plane electrical resistivity was measured using a four-contact configuration and a low-frequency ac-resistance bridge. Both experiments were performed in a commercial multifunctional equipment. Zero field cooling dc-magnetic susceptibility was carried out through a commercial Superconducting Quantum Interference Device (SQUID)/platform with magnetic field of $1.5 \mathrm{~T}$ applied parallel to the ab-plane. Single crystal synchrotron x-ray diffraction experiments were performed at the XDS beamline of the Brazilian Synchrotron Light Laboratory (LNLS) [45]. A sagittal focusing double $\mathrm{Si}(111)$ crystal monochromator and Rh-coated vertically collimating and focusing mirrors were employed as optical elements of the beamline. The selected wavelength was $\lambda=0.6196 \AA$, calibrated with a powder diffraction scan of a $\mathrm{LaB}_{6}$ NIST standard. The beam dimensions were $\sim 0.2(\mathrm{~V}) \times 2(\mathrm{H}) \mathrm{mm}^{2}$ at the sample position. A $10 \mathrm{~K}$ closed-cycle He cryostat holding the crystals was mounted in the Eulerian cradle of a $6+2$ circle diffractometer. The diffracted beam was detected using a setup attached to the $2 \theta$ arm of the diffractometer, including a scattering slit close to the sample, a vertical resolution slit of $1 \mathrm{~mm}$ placed at $\sim 1 \mathrm{~m}$ from the sample and a high-throughput LaBr scintillator point detector. The lattice parameters are $a=3.933(1)$
$\AA$ and $c=12.254(3) \AA$ (SG: $I 4 / \mathrm{mmm}$ ) at $T=165$ $\mathrm{K}$. The strain distribution along $c$, determined from the width of the $(00 l)$ Bragg reflections, is $\Delta c / c=0.03$. Raman scattering experiments were performed in quasibackscattering geometry with an $\mathrm{Ar}^{+} 488 \mathrm{~nm}$ exciting laser line and a $1800 \mathrm{~mm}^{-1}$ triple grating spectrometer equipped with a $L \mathrm{~N}_{2}$-cooled multichannel CCD detector. A fresh $a b$ surface was obtained by cleavage, being promptly loaded onto the cold finger of a closed-cycle He cryostat with base temperature of $\sim 20 \mathrm{~K}$. All spectra were taken with $\mathrm{a} \sim 4 \mathrm{~mW}$ laser power and a corresponding correction of $0.5 \mathrm{~K} / \mathrm{mW}$ for local heating was applied for each spectrum $[31,35]$. A constant intensity offset $D$ unrelated with the Raman process was subtracted from the raw intensities to extract the Raman signal such as described in Ref. 35 and the same crystal of SFCA was used for all experiments reported in this work. The same side of the sample was probed by XRD and Raman measurements and all the temperaturedependent experiments were performed on cooling.

\section{RESULTS AND ANALYSIS}

\section{A. Specific heat, resistivity and magnetic susceptibility}

Figure 1(a) shows the in-plane resistivity $(\rho)$ curve. The inset shows the specific heat $\left(C_{p}\right)$ and resistivity slope $(\mathrm{d} \rho / \mathrm{d} T)$ in a narrow $T$ interval. Besides the superconducting transition at $T_{c}=22 \mathrm{~K}$, two inflection points are clearly observed from the resistivity data. These transition temperatures are also evident in specific heat data. Such temperatures seem to delimit an intermediate state where the specific heat remains at higher values with respect to the baselines below $132 \mathrm{~K}$ and above $152 \mathrm{~K}$. Figure 1(b) shows that magnetic susceptibility also displays a small anomaly in the temperature interval between the transitions delimited by specific heat and resistivity data, presumably marking the onset of a spin density wave order. These data suggest that below $\sim 132 \mathrm{~K}$ this material is in a homogeneous long-range ordered magnetic state, although further experiments such as neutron diffraction would be necessary to confirm this hypothesis unambiguously. From these experiments, we did not observe latent heat at either transition temperatures [46].

\section{B. X-ray diffraction}

Figure 2 shows a contour plot of $(h h l=20)$ reciprocal space scans around $h=2$ between $T=110$ and 161 $\mathrm{K}$ in the following sequence of temperatures: $160.8 \mathrm{~K}$, $151.7 \mathrm{~K}, 147.9 \mathrm{~K}, 145.5 \mathrm{~K}, 140.8 \mathrm{~K}, 132.8 \mathrm{~K}, 131.8 \mathrm{~K}$, $124.6 \mathrm{~K}, 114.6 \mathrm{~K}, 110.8 \mathrm{~K}$. The reciprocal space indexing is defined with respect to the tetragonal cell at $T=161$ $K$. Notice that this Bragg reflection is relatively broad. This is due to lattice strain that may arise from micro-

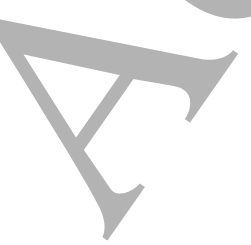



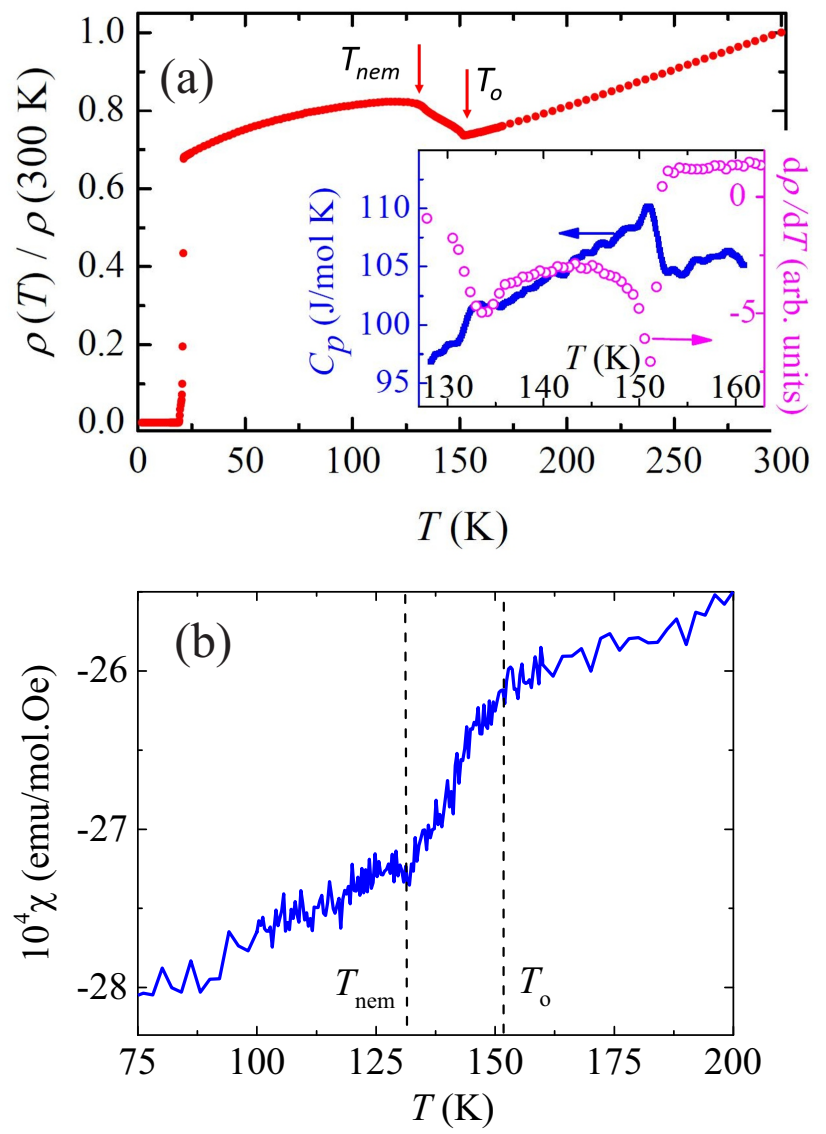

FIG. 1: (a) In-plane resistivity of $\operatorname{Sr}\left(\mathrm{Fe}_{1-x} \mathrm{Co}_{x}\right)_{2} \mathrm{As}_{2} \sqrt{\mathrm{x}}$ $=0.24(3)]$. The inset shows the specific heat $C_{p}$ and the resistivity slope in a selected temperature interval. (b) $T$ dependency of the dc molar magnetic susceptibility. The red arrows and dashed lines set the defined temperatures $T_{n e m}$ and $T_{o}$.

scopic variations of the Co-content along the crystal and other possible structural defects, which lead to a distribution of lattice parameters and a consequent blurring of the reciprocal-space scans. This effect seriously limits our capacity to investigate in detail the lattice parameters changes as a function of temperature. Nonetheless, it is still possible to detect relevant changes in the crystal structure in the studied temperature interval, which seem to be associated with the transitions captured by specific heat, resistivity and susceptibility data (see above). At $\sim 160 \mathrm{~K}$, the diffraction profile is associated with the $(22$ 20) reflection of a pure tetragonal phase. Below $T_{o} \sim 152$ $\mathrm{K}$, this profile starts to evolve, with the appearance of a significant intensity at $h \sim 2.01$ and reduction of the intensity of the main peak at $h \sim 2.00$. This trend becomes more accentuated for this crystal below $\sim 140 \mathrm{~K}$, and below $\sim 132 \mathrm{~K}$. the tetragonal peak disappears. At $\sim 110$ $\mathrm{K}$, it is possible to identify two separated maxima that we ascribe to the orthorhombic (4 0 20) and (0 4 20) reflections. The unequal intensities of these reflections are

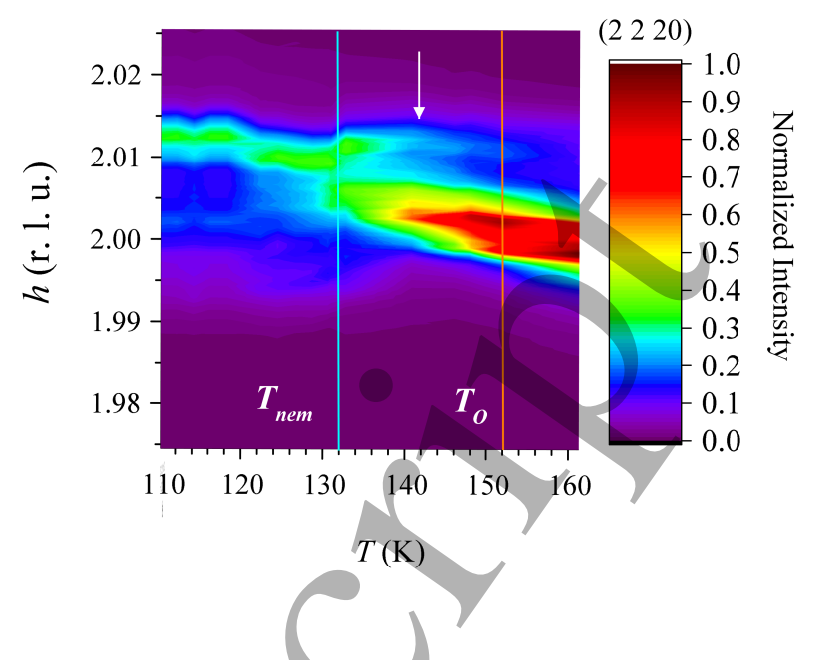

FIG. 2: Contour plots of the x-ray scattering around the $(h h$ 20) reflection (tetragonal axes). The transition temperatures $T_{n e m}$ and $T_{o}$ corresponding to features in specific heat and resistivity data are indicated as vertical lines [see also Fig. 1]. The white arrow marks an structural anomaly for this crystal at $\sim 140 \mathrm{~K}$. In the color scale, the intensities were divided by a global factor of $9 \times 10^{4}$ counts (maximum intensity at 160.8 $\mathrm{K})$.

not necessarily associated with uneven population of the orthorhombic domains, since such intensities are highly sensitive to the alignment procedure, which could not be simultaneously optimized for both reflections. We conclude that, despite the inherent disorder brought by the large Co substitution in this compound, we can unequivocally identify the structural phases below $\sim 132 \mathrm{~K}$ and above $T_{o}$ as purely orthorhombic and tetragonal, respectively, and that a coexistence of orthorhombic and tetragonal domains takes place between these temperatures.

\section{Raman scattering}

In this work a planar 2 Fe unit cell [see Fig. 3] is used to described the phonon and electronic symmetries. For this configuration, the crossed polarization $X Y$ are aligned with the diagonals of the Fe-Fe bonds and it probes the $B_{2 g}$ symmetry, rotating the polarizers by $45^{\circ}$, in the $X^{\prime} Y^{\prime}$ polarization, $B_{1 g}$ symmetry is selected. However, it is worth mentioning that for a 1 Fe unit cell, with axes aligned to the Fe-Fe bonds, crossed polarizations XY and X'Y' probe $B_{1 g}$ and $B_{2 g}$ symmetries, respectively. Thus, what is $B_{2 g}\left(B_{1 g}\right)$ in $2 \mathrm{Fe}$ unit cell is $B_{1 g}\left(\mathrm{~B}_{2 g}\right)$ in $1 \mathrm{Fe}$ unit cell [30]. From a charge/orbital nematic fluctuations point of view (in $2 \mathrm{Fe}$ unit cell) both $B_{2 g}$ and $B_{1 g}$ symmetries are allowed, although the latter was not observed in iron-pnictides up to our knowledge [29]. For a spin nematic fluctuations scenario, only $B_{2 g}(1 \mathrm{Fe}$ unit cell) $\left[B_{1 g}(2 \mathrm{Fe}\right.$ unit cell $\left.)\right]$ symmetry is fully cancelled, being both interpretations consistent [31]. 
Figures 3(a) and 3(b) show the tetragonal 2 Fe unit cell with the defined $X Y$ and $Y Y$ polarizations and the associated Raman spectra at $T=290 \mathrm{~K}$, repectively. The $X Y(Y Y)$ polarization selects the $B_{2 g}\left(A_{1 g}+B_{1 g}\right)$ symmetry in the tetragonal phase and $A_{g}\left(A_{g}+B_{1 g}\right)$ in the orthohombic phase. Raman-active phonon modes are expected for orthorhombic $A_{g}$, and tetragonal $A_{1 g}$ and $B_{1 g}$ symmetries, whereas no phonon is expected in tetragonal $B_{2 g}$ symmetry [47]. Instead, this is the symmetry where electronic nematic fluctuations were unambiguously observed in several similar compounds [29-31, 35]. Thus, at room temperature only a flat electronic Raman spectrum is expected at the $B_{2 g}$ symmetry in $X Y$ polarization, while a $B_{1 g}$ Fe phonon $\left(\sim 208 \mathrm{~cm}^{-1}\right)$ is observed in $Y Y$ polarization, as confirmed in Fig. 3(b).

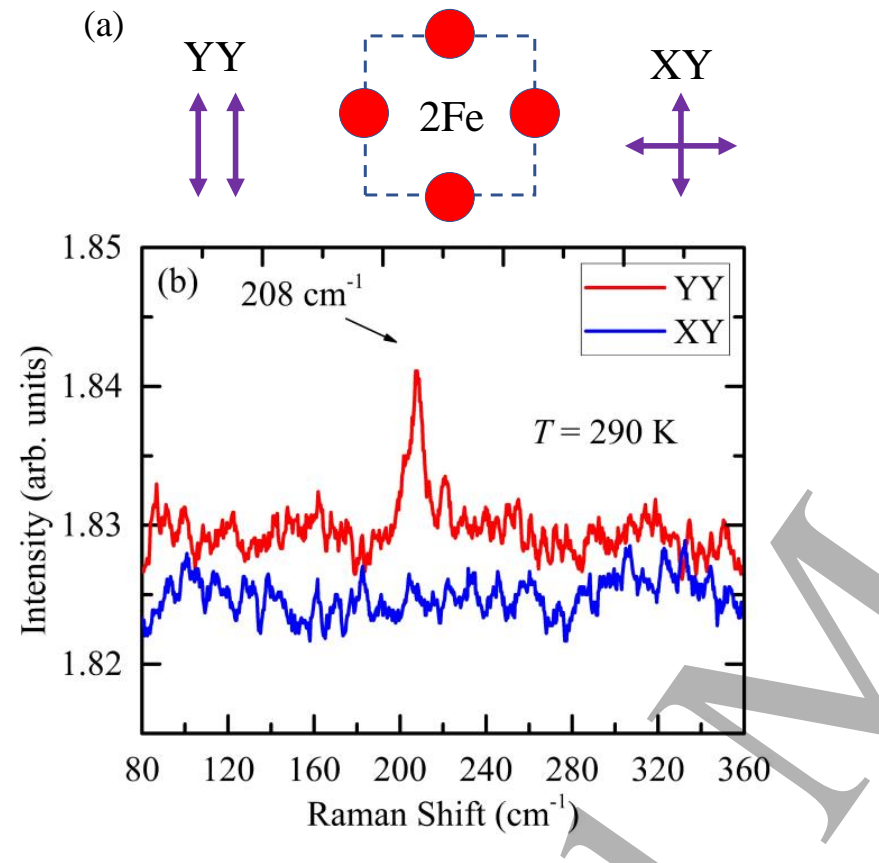

FIG. 3: (Color online) (a) Tetragonal 2Fe square unit cell and adopted $Y Y$ and $X Y$ polarizations. The arrows indicate the polarizations of incoming and outgoing radiation (b) Raman spectra at $290 \mathrm{~K}$ in $Y Y$ polarization (red) showing the $\mathrm{B}_{1 g}$ Fe phonon and $X Y$ polarization (blue) with a $\mathrm{B}_{2 g}$ symmetry.

The raw Raman intensity $I(\omega, T)$ is related to the imaginary part of the Raman response $\chi^{\prime \prime}(\omega, T)$ through the fluctuation-dissipation theorem that gives $I(\omega, T)=$ $(1+n) \chi^{\prime \prime}(\omega, T)$, with the Bose-Einstein statistical factor $n \equiv 1 /\left(e^{\hbar \omega / k_{B} T}-1\right)[35]$. Figure 4(a) shows the raw Raman spectra for selected temperatures, whereas in Figs. $4(\mathrm{~b})$ and $4(\mathrm{c}),\left[\chi^{\prime \prime}(\omega, T) / \omega\right]$ spectra in $X Y$ polarization with a Quasi-Elastic Peak (QEP) profile for selected temperatures are shown. The Raman conductivity is fitted by $\chi_{B_{2 g}}^{\prime \prime}(\omega, T) / \omega=A(T) \Gamma(T) /\left(\omega^{2}+\Gamma(T)^{2}\right)+b$, where $A(T)$ and $\Gamma(T)$ are the area and width of the QEP, respectively, and $b$ is a contribution that might not be directly related with nematic fluctuations, likely represent-

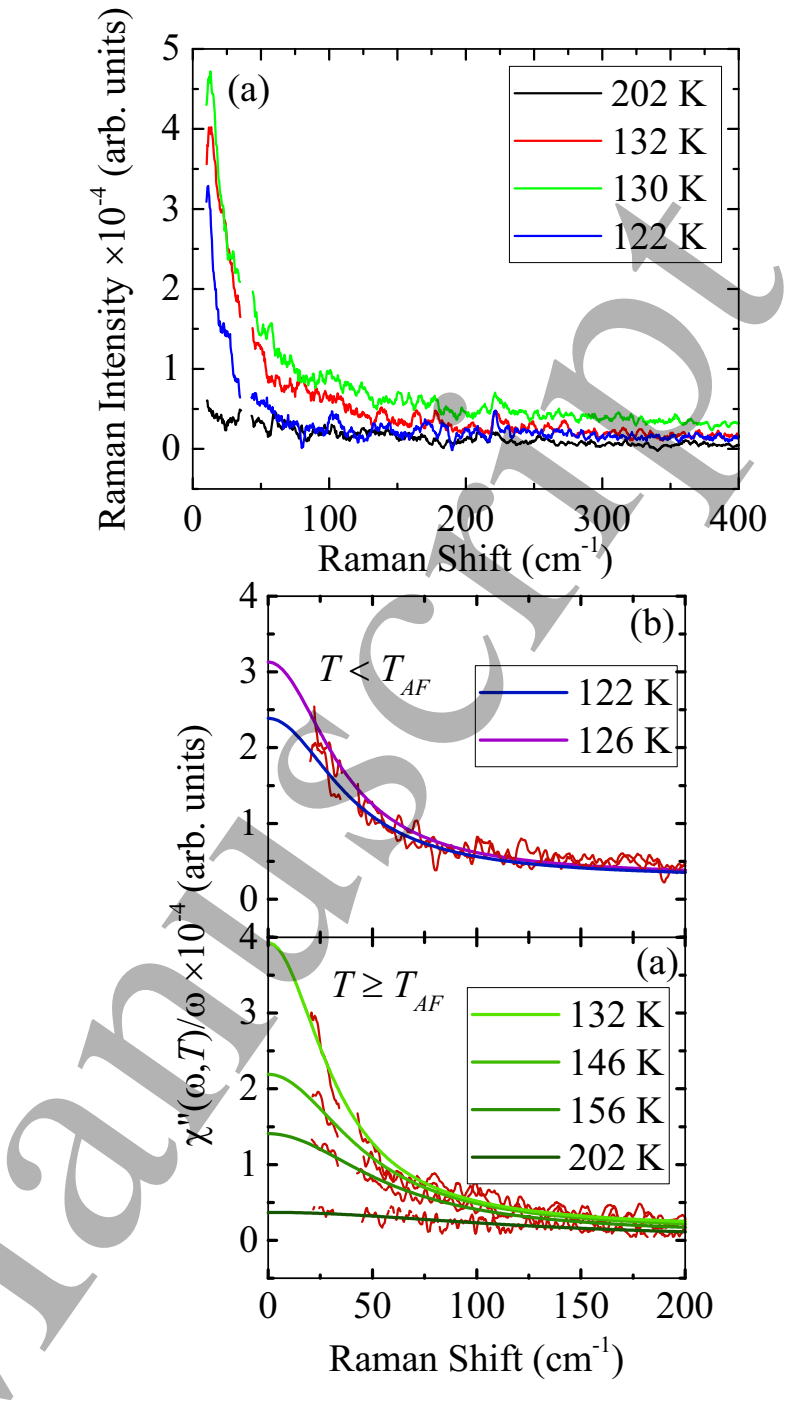

FIG. 4: (Color online) (a) Selected raw Raman spectra for selected temperatures. $T$-dependence of $\chi^{\prime \prime}(\omega, T) / \omega(\mathrm{b})$ below and (c) above $T_{n e m}$. The solid lines are the Lorentzian fittings centered at zero frequency and a spurious peak at $38 \mathrm{~cm}^{-1}$ due to the laser plasma line was removed from the experimental data.

ing the low-frequency limit of two-magnon scattering that peaks at much higher frequencies $[35,48,49]$. The detailed $T$-dependence of $A, \Gamma$ and height $A / \Gamma$ of the QEP are shown in Figs. 5(a), 5(b) and 5(c), respectively. Here, only data between $\sim 120$ and $160 \mathrm{~K}$ are shown, where the QEP signal is sufficiently strong to yield reliable fitting parameters. Maxima are observed in both QEP area and height around $\sim 132 \mathrm{~K}$, and the QEP height shows no visible anomaly at $T_{o}$. On the other hand, the QEP width appears to show. anomalies in the temperature range where the orthorhombic domains develop [see Fig. 5(c)]. Particularly, appreciable QEP sharpenings are observed below 155 and $140 \mathrm{~K}$, corresponding approximately to the temperatures where the orthorhombic domains starts 


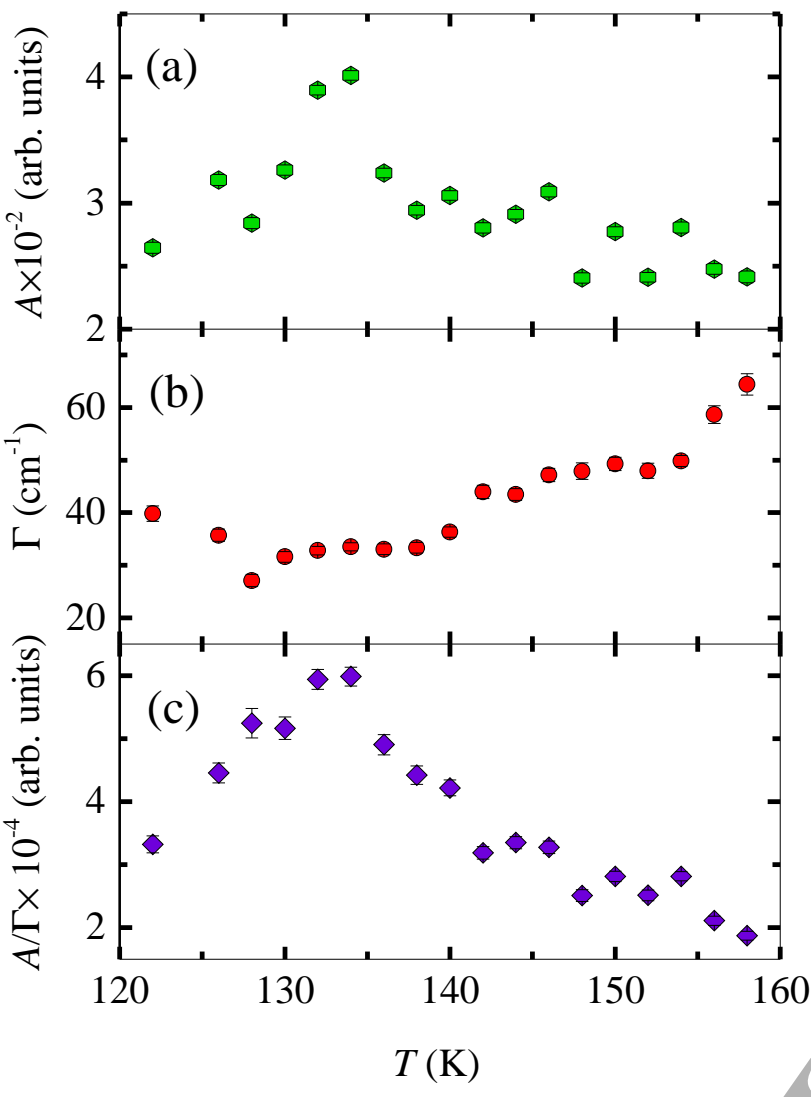

FIG. 5: (Color online) $T$-dependence of (a) area $A$, (b) width $\Gamma$ and (c) height $A / \Gamma$ of $B_{2 g}$ QEP (2Fe tetragonal cell). Error bars, when not visible, are smaller than the symbol sizes.

to be observed $\left(T_{o}\right)$ and where they experience a clear strengthening, respectively, according to XRD data (see Fig. 2).

\section{DISCUSSION}

As mentioned above, the ubiquitous QEP found in $B_{2 g}$ symmetry in parent compounds of Fe-based superconductors has been ascribed to electronic nematic fluctuations. Different detailed mechanisms have been proposed to explain the origin of this Raman activity, evoking either the magnetic or charge/orbital degrees of freedom, and in principle both mechanisms may be at play, possibly with different relative cross sections for each material. In any case, it is safe to state that the temperature where the QEP reaches its maximum intensity marks the electronic nematic transition temperature, i.e., $T_{n e m} \sim 132$ $\mathrm{K}$ for this compound. It is interesting to note that a peak is observed in the specific heat data at this temperature, accompanied by changes in the resistivity and magnetic susceptibility (see Fig. 1)

Kretzschmar et al. showed that, for lightly Co-doped $\mathrm{BaFe}_{2} \mathrm{As}_{2}$, the QEP height has a maximum value at the orthorhombic-tetragonal transition temperature $T_{o}$, thus $T_{n e m}=T_{o}$ for that system [31]. Also, Massat et al. showed that, for FeSe, the maximum intensity of the QEP coincides with the $T_{o}$ and also with the temperature below which substantial electronic anisotropy has been observed by transport measurements [36]. In fact, while the non-equality of magnetic ordering and nematic transition temperatures is a well known fact for Fe pnictide physics, it is generally assumed that $T_{o}$ and $T_{n e m}$ are the same temperature. However, as shown below, this is not necessarily the case. While stripe magnetism, charge anisotropy and orthorhombicity are clearly interconnected in this system, they represent different degrees of freedom, which, at least in principle, may show different critical temperatures.

For our sample of SFCA, the QEP area and height show maxima at $T_{\text {nem }}$ [Figs. $5(\mathrm{a})$ and $5(\mathrm{c})$ ] with no observable anomaly at $T_{o}$. This indicates that the nematic transition is not anchored to the onset of the orthorhombic phase in this compound. On the other hand, the QEP width shows substantial anomalies at $\sim 155$ and $140 \mathrm{~K}$ [Fig. 5(b)]. According to our synchrotron XRD data performed in the same crystal of the Raman study [Fig. 2], these temperatures seem to coincide with structural anomalies, namely the onset of orthorhombic domains at $T_{o} \sim 152 \mathrm{~K}$ and the strengthening of such domains below $\sim 140 \mathrm{~K}$. Our specific heat, resistivity and magnetic susceptibility data indicate marked transitions at $T_{o}=152 \mathrm{~K}$, presumably corresponding to the onset of the orthorhombic state, and around $\sim 132 \mathrm{~K}$ that is the nematic transition. Despite these data do not show any marked peak at $\sim 140 \mathrm{~K}$, ripples are observed in specific heat between $T_{o}$ and $T_{n e m}$, which might be the manifestation of the Co-concentration distribution in this sample.

In principle, the fact that $T_{n e m}$, defined as the QEP maximum intensity, does not coincide with $T_{o}$, i.e., the onset of orthorhombic domains, might be ascribed to the structurally phase-separated state between $T_{n e m}$ and $T_{o}$ revealed by our XRD data (Fig. 2). This highly disordered state is also manifest in our specific heat, resistivity and magnetic susceptibility data. In this tentative scenario, each domain would have its own local $T_{n e m} \sim T_{o}$, and the increasing QEP signal observed on cooling below $T_{o}$ would be mostly originated at the reminiscent tetragonal / electronically disordered domains. This was indeed the interpretation given to explain a similar result for a LaFeAsO crystal [35]. On the other hand, if the increasing Raman QEP on cooling down to $T_{n e m}$ is dominated by the signal arising from the tetragonal domains, the anomalies in the QEP width at $T_{o}$ and $\sim 140$ $\mathrm{K}$ are not trivially understood. This is because the QEP sharpening is indicative of an abrupt reduction of the average nematic fluctuation rate, whereas the QEP lineshape arising from the tetragonal domains alone is expected to be insensitive to the appearance and growth of 
the orthorhombic domains.

The above considerations associated with the large Co-content distribution through the sample, lead us to propose the alternative scenario where the orthorhombic domains immediately below $T_{o}$ are also in an electronically disordered state, and still show increasing contribution to the QEP signal on cooling, with a global nematic transition temperature $T_{n e m}$. In this scenario, the orthorhombic domains show slower nematic fluctuations than the coexisting tetragonal domains, explaining why the domain-average QEP width shows anomalies at temperatures where the balance between orthorhombic and tetragonal domains is significantly changed. We should mention that an anomaly of the QEP width at $T_{o}$ was also observed for $\mathrm{LaFeAsO}$, which exhibit large separation between magnetic and structural transition temperatures, indicating that the proposed scenario may be extrapolated to other materials with similar property or with high dopant-content distribution as in SFCA.

A scenario where the orthorhombic transition is not coincident with either the charge or spin stripe transition temperatures thus deserves further consideration. It is clear that an orthorhombic structure is expected under either the charge or spin stripe state, since both phases break the four-fold rotational symmetry. On the other hand, no symmetry argument dismisses the possibility of the orthorhombic state be developed above both the magnetic and charge/orbital transition temperatures.

The electron-phonon interaction that couples the nematic fluctuations to local orthorhombic distortions at high temperatures is expected to be strongly dependent on the time scale of the former compared to the typical phonon period, which sets the time scale for atomic motion. In fact, if the nematic fluctuation rate is significantly lower than the relevant phonon frequency, the nuclei positions will have sufficient time to respond adiabatically to the electronic fluctuations, leading to accompanying local orthorhombic fluctuations and strain. In the opposite extreme, electronic nematic fluctuations that are faster than the phonon timescale will not be able to cause substantial local orthorhombic distortions. For SFCA and $\mathrm{LaFeAsO}$, the nematic fluctuation rate is in the range $\Gamma<50 \mathrm{~cm}^{-1}$ for $T<T_{\text {nem }}+20$ $K$. This $\Gamma$ is substantially lower than the typical optical phonon frequency range $\left(\omega \gtrsim 150 \mathrm{~cm}^{-1}\right)$. It is thus reasonable to consider that a quasi-adiabatic electronic/lattice fluctuation regime is achieved in this temperature range and local orthorhombic clusters follow the nematic fluctuations even at temperatures substantially above $T_{n e m}$. For sufficiently dense concentrations of dynamic orthorhombic clusters, a percolation limit will be presumably achieved that will sustain long-range phase coherence in a diffraction process, leading to the formation of long-range (albeit still dynamical) orthorhombic microdomains for $T_{n e m}<T<T_{o}$. This highly dis- ordered electronic/lattice state would further evolve on cooling and transform into a long-range-ordered and stable charge and/or spin stripe state for $T<T_{\text {nem }}$.

It is interesting to note that, while $\mathrm{LaFeAsO}$ and SFCA show largely separated $T_{o}$ and $T_{n e m}$, such separation is much smaller or non-existent for $\mathrm{BaFe}_{2} \mathrm{As}_{2}$. This is naturally explained within our picture, since the QEP width $\Gamma \sim 100 \mathrm{~cm}^{-1}$ for the latter compound at $\sim T_{n e m}+20 \mathrm{~K}$ $\sim 160 \mathrm{~K}$, which is much higher than the values observed for $\mathrm{LaFeAsO}$ and SFCA at a similar temperature scale. The much faster nematic fluctuations for $\mathrm{BaFe}_{2} \mathrm{As}_{2}$ may be sufficient to prevent the system to enter into the adiabatic regime above $T_{n e m}$, and the orthorhombic phase transition occurs only at $T_{o} \sim T_{n e m}$. However, in the $\mathrm{K}$-doped $\mathrm{BaFe}_{2} \mathrm{As}_{2}$ system, in which magnetic and structural transition temperatures occur $\sim 80 \mathrm{~K}, \Gamma \sim 50 \mathrm{~cm}^{-1}$ for $\sim T_{\text {nem }}+20 \mathrm{~K} \sim 100 \mathrm{~K}$ [33]. In this compound, $\mathrm{K}$ substitutes $\mathrm{Ba}$ instead $\mathrm{Fe}$, which might be another ingredient to decrease the relaxation rate in Fe-pnictides and should be better investigated.

\section{CONCLUSIONS}

In summary, a coexistence of long-range orthorhombic and tetragonal domains in $\operatorname{Sr}\left(\mathrm{Fe}_{1-x} \mathrm{Co}_{x}\right)_{2} \mathrm{As}_{2}[x=$ $0.24(3)]$ was observed by x-ray diffraction between the critical temperatures $T_{n e m}=132 \mathrm{~K}$ and $T_{o}=152 \mathrm{~K}$ marked by specific heat, resistivity, magnetic susceptibility data. A temperature-dependent QEP signal associated with electronic nematic fluctuations was observed in the electronic Raman $\mathrm{B}_{2 g}$ channel with area and height maxima marking the nematic transition temperature around $T_{n e m} \sim 132 \mathrm{~K}$. Sharpenings of the QEP width below $\sim T_{o}$ and $\sim 140 \mathrm{~K}$ are associated with the onset of orthorhombic domains and its enhancement, respectively. Relatively slow (compared to the optical phonons timescale) nematic fluctuations for $T \gtrsim T_{n e m}$, such as found here in SCFA and also in LaFeAsO [35], favors the formation of dynamical orthorhombic clusters following the electronic nematic correlations, which may become sufficiently dense to sustain long-range phase coherence in a diffraction process at temperatures substantially above $T_{n e m}$.

\section{Acknowledgments}

We thank M.A. Eleotério for assistance in conducting the x-ray diffraction experiments. LNLS is acknowledged for concession of beamtime. This work was supported by FAPESP Grants 2017/10581-1, 2012/05906-3, and 2015/15665-3, and CNPq, Brazil. 
[1] Y. Kamihara, T. Watanabe, M. Hirano and H. Hosono, J. Am. Chem. Soc. 130, 3296 (2008).

[2] M. S. Torikachvili,S. L. Budko, N. Ni, and P. C. Canfield, Phys. Rev. Lett. 101, 057006 (2008).

[3] B. Lv, L. Deng, M. Gooch, F. Wei, Y. Sun, J. K. Meen, Y. -Y. Xue, B. Lorenz, and C.-W. Chu, Proc. Natl. Acad. Sci. USA 108, 15705 (2011).

[4] P. L. Alireza, Y. C. Ko, J. Gillett, C. M. Petrone, J. M. Cole, G. G.Lonzarich and S. E. Sebastian, J. Phys.: Condens. Matter 21, 012208 (2008).

[5] T. Garitezi, C. Adriano, P. Rosa, E. Bittar, L. Bufaical, R. de Almeida, E. Granado, E. Grant, Z. Fisk and M Avila. Braz. J. Phys. 43, 223 (2013).

[6] A. Leithe-Jasper, W. Schnelle, C. Geibel and H. Rosner, Phys. Rev. Lett. 101, 207004 (2008).

[7] F.-C. Hsu, J.-Y. Luo, K.-W. Yeh, T.-K. Chen, T.-W. Huang, P.-M. Wu, Y.-C. Lee, Y.-L, Huang, Y.-Y. Chu, D.-C. Yan, Proc. Natl. Acad. Sci. USA 105, 14262 (2008).

[8] R. Fernandes, A. Chubukov, J. Schmalian, Nat. Phys. 10, 97 (2014).

[9] J.-H. Chu, J. G. Analytis, K. De-Greve, P. L. McMahon, Z. Islam, Y. Yamamoto and I. R. Fisher, Science 329, $824(2010)$.

[10] S. Kasahara, H. Shi, K. Hashimoto, S. Tonegawa, Y. Mizukami, T. Shibauchi, K. Sugimoto, T. Fukuda, T. Terashima, A. H. Nevidomskyy, Nature 486, 382 (2012).

[11] M. Yi, D. Lu, J.-H. Chu, J. G. Analytis, A. P. Sorini, A. F. Kemper, B. Moritz, S.-K. Mo, R. G. Moore, M. Hashimoto, Proc. Natl. Acad. Sci. USA 108, 6878 (2011)

[12] M. Nakajima, T. Liang, S. Ishida, Y. Tomioka, K. Kihou, C. Lee, A. Iyo, H. Eisaki, T. Kakeshita, T. Ito, Proc Natl. Acad. Sci. USA 108, 12238 (2011).

[13] P.F.S. Rosa, C. Adriano, T.M. Garitezi, T. Grant, Z. Fisk, R.R. Urbano and P.G. Pagliuso, Sci. Rep. 4, 6543 (2014).

[14] H.-H. Kuo, J.-H. Chu, J. C. Palmstrom, S. A. Kivelson and I. R. Fisher, Science 352, (2016).

[15] R. M. Fernandes and J. Schmalian, Supercond. Sci. Technol. 25, 084005 (2012).

[16] M. Kim, R. M. Fernandes, A. Kreyssig, J. W. Kim, A. Thaler, S. L. Budko, P. C. Canfield, R. J. McQueeney, J. Schmalian, and A. I. Goldman, Phys. Rev. B 83, 134522 (2011).

[17] D. Pratt, W. Tian, A. Kreyssig, J. Zarestky, S. Nandi, N. Ni, S. Budko, P. Canfield, A. Goldman and R. McQueeney, Phys. Rev. Lett. 103, 087001 (2009).

[18] M. Piva, M. Besser, K. Mydeen, T. Garitezi, P. Rosa, C. Adriano, T. Grant, Z. Fisk, R. Urbano, M. Nicklas and P. G. Pagliuso, J. Phys.: Condens. Matter 27, 145701 (2015).

[19] V. K. Thorsmølle, M. Khodas, Z.-P. Yin, C. Zhang, S. V. Carr, P. Dai and G.Blumberg, Phys. Rev. B 93, 054515 (2016).

[20] F. Steckel, M. Roslova, R. Beck, I. Morozov, S. Aswartham, D. Evtushinsky, C. G. Blum, M. AbdelHafiez, D. Bombor, J. Maletz, Phys. Rev. B 91, 184516 (2015).

[21] J. Zhao, Q. Huang, C. De La Cruz, S. Li, J. Lynn, Y. Chen, M. Green, G. Chen, G. Li, Z. Li, Nat. Mater.7, 953 (2008).
[22] J. Zhao, Q. Huang, C. de La Cruz, J. Lynn, M. Lumsden, Z. Ren, J. Yang, X. Shen, X. Dong, Z. Zhao, Phys. Rev. B 78, 132504 (2008).

[23] Y. Qiu, W. Bao, Q. Huang, T. Yildirim, J/Simmons, M. Green, J. Lynn, Y. Gasparovic, J. Li, T. Wu, Phys. Rev. Lett. 101, 257002 (2008).

[24] Y. Chen, J. Lynn, J. Li, G. Li, G. Chen, J. Luo, N. Wang, P. Dai, C. de la Cruz and H. Mook, Phys. Rev. B 78, 064515 (2008).

[25] M. A. McGuire, R. P. Hermann, A. S. Sefat, B. C. Sales, R. Jin, D. Mandrus F. Grandjean and G. J. Long, New J. Phys. 11, 025011 (2009).

[26] H.-F. Li, W. Tian, J.-Q. Yan, J. Zarestky, R. McCallum, T. Lograsso and D. Vaknin, Phys., Rev. B 82, 064409 (2010).

[27] L. Chauvière, Y. Gallais, M. Cazayous, M. A. Measson, A. Sacuto, D. Colson and A. Forget, Phys. Rev. B 82, 180521 (2010).

[28] L. Chauvière, Y. Gallais, M. Cazayous, M. Méasson, A. Sacuto, D. Colson, and A. Forget, Phys. Rev. B 84, 104508 (2011).

[29] Y. Gallais, R. Fernandes, I. Paul, L. Chauviere, Y.-X. Yang, M. A. Méasson, M. Cazayous, A.Sacuto, D. Colson and A. Forget, Phys. Rev. Lett. 111, 267001 (2013).

[30] Y. Gallais and I. Paul, C. R. Phys. 17, 113 (2016).

[31] F. Kretzschmar, T. Böhm, U. Karahasanović, B. Muschler, A. Baum, D. Jost, J. Schmalian, S. Caprara, M. Grilli, C. Di-Castro, Nat. Phys. 12, 560 (2016).

[32] W.-L. Zhang, Z. Yin, A. Ignatov, Z. Bukowski, J. Karpinski, A. S. Sefat, H. Ding, P. Richard, and G. Blumberg, Phys. Rev. B 93, 205106 (2016).

[33] T. Böhm, R. Hosseinian Ahangharnejhad, D. Jost, A. Baum, B. Muschler, F. Kretzschmar, P. Adelmann, T. Wolf, H.-H. Wen, J.-H. Chu, Phys. Status Solidi B 254, 1600308 (2017).

[34] S.-F. Wu, P. Richard, H. Ding, H.-H. Wen, G. Tan, M. Wang, C. Zhang, P. Dai and G. Blumberg, Phys. Rev. B 95, 085125 (2017).

[35] U. Kaneko, P. Gomes, A. García-Flores, J.-Q. Yan, T. Lograsso, G. Barberis, D. Vaknin and E. Granado, Phys. Rev. B 96, 014506 (2017).

[36] P. Massat, D. Farina, I. Paul, S. Karlsson, P. Strobel, P. Toulemonde, M. A. Méasson, M. Cazayous, A. Sacuto, S. Kasahara, A. Sacuto, S. Kasahara, T. Shibauchi, Y. Matsuda, and Y. Gallais, Proc. Natl. Acad. Sci. USA 13 (33), 9177 (2016).

[37] V. Gnezdilov, Y. G. Pashkevich, P. Lemmens, D. Wulferding, T. Shevtsova, A.Gusev, D. Chareev and A. Vasiliev, Phys. Rev. B 87, 144508 (2013).

[38] H. Yamase, R. Zeyher, Phys. Rev. B 88, 125120 (2013).

[39] U. Karahasanovic, F. Kretzschmar, T. Böhm, R. Hackl, I. Paul, Y. Gallais and J. Schmalian, Phys. Rev. B 92, 075134 (2015).

[40] M. Khodas and A. Levchenko, Phys. Rev. B 91, 235119 (2015).

[41] H. Yamase and R. Zeyher, New J. Phys. 17, 073030 (2015).

[42] A. Leithe-Jasper, W. Schnelle, C. Geibel, and H. Rosner, Phys. Rev. Lett. 101, 207004 (2008).

[43] J. S. Kim, S. Khim, H. J. Kim, M. J. Eom, J. M. Law, R. K. Kremer, J. H. Shim and K. H. Kim, Phys. Rev. B 
82, 024510 (2010).

[44] E. Lengyel, M. Kumar, W. Schnelle, A. Leithe-Jasper and M. Nicklas, Phys. Status Solidi B, 254, 1600154 (2016).

[45] F. Lima, M. Saleta, R. Pagliuca, M. Eleotério, R. Reis, J. Fonseca Júnior, B. Meyer, E. Bittar, N. Souza-Neto and E. Granado, Journal of Synchrotron Radiation 23 (2016).

[46] J.C.Lashley, M.F.Hundley, A.Migliori, J.L.Sarrao, P.G.Pagliuso, T.W.Darling, M.Jaime, J.C.Cooley, W.L.Hults, L.Morales, D.J.Thoma, J.L.Smith, J.BoerioGoates, B.F.Woodfield, G.R.Stewart, R.A.Fisher, N.E.Phillips, Cryogenics 43, 369 (2003).
[47] V. Hadjiev, M. Iliev, K. Sasmal, Y.-Y. Sun, and C. Chu, Phys. Rev. B 77, 220505 (2008).

[48] S. Sugai, Y. Mizuno, K. Kiho, M. Nakajima, C. Lee, A. Iyo, H. Eisaki and S. Uchida, Phys. Rev. B82, 140504 (2010).

[49] S. Sugai, Y. Mizuno, R. Watanabe, T. Kawaguchi, K. Takenaka, H. Ikuta, K. Kiho, M. Nakajima, C. Lee, A. Iyo, J Supercond. Nov. Magn. 26, 1179 (2013).

[50] R. Hu, S. L. Budko, W. E. Straszheim and P. C. Canfield, Phys. Rev. B 83, 094520 (2011). 